\title{
Principi scientifici e soddisfazione del paziente: due cose importanti da non dimenticare nella gestione degli accessi vascolari
}

\author{
Sandro Geatti
}

\section{Rappresentante Nazionale EDTNA-ERCA}

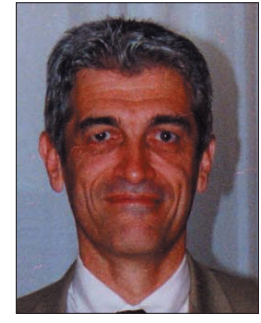

$\mathbf{T}$ utti gli infermieri che operano in dialisi devono presto o tardi misurarsi con situazioni più o meno critiche legate alla gestione dell'accesso vascolare del paziente: messa in crisi della propria abilità, conflitti più o meno aspri con il paziente, richieste di aiuto al medico.

L'efficacia depurativa della dialisi dipende in maniera preponderante dalla qualità dell'accesso, il cui mantenimento in buona salute dovrebbe rappresentare una vera e propria "mission" per tutto il personale sanitario del team nefrologico.

Da un punto di vista infermieristico ritengo che i parametri di riferimento da tenere in considerazione per una gestione "al meglio" dell'accesso vascolare siano i seguenti:

- Metodologia di approccio.

- Punto di vista del paziente.

La metodologia di approccio altra non può essere se non scientifica, cioè basata sull'evidenza, che nel ca- so specifico si traduce nell'assunzione di specifici protocolli operativi quali strumenti di integrazione organizzativa.

Gli infermieri dovrebbero essere in grado di utilizzarli in forma "attiva", partecipata e soprattutto condivisa, dalla costruzione (basata su linee guida, se esistenti, e su una revisione puntuale della letteratura) alla sperimentazione, alla valutazione dell'efficacia dello strumento, alla sua periodica revisione.

Si tratta di un processo che deve trovare idonei momenti di condivisione anche con il medico, soprattutto negli aspetti a carattere prescrittivo, sul $\mathrm{co}^{-}$ me, sul quando e sul perché.

Nell'applicare i protocolli bisogna tener presente anche il livello di competenza individuale, che è correlato al fattore sicurezza-qualità e all'assunzione di responsabilità. Ciò significa che la performance massima, a esempio nella procedura di puntura-incannulazione della $\mathrm{FAV}$, non potrà essere assicurata, in tutte le situazioni-condizioni esistenti, a diverso grado di complessità, da tutti gli infermieri.

$\mathrm{Si}$ prospetta quindi l'individuazione della figura dell" "infermiere esperto" cioè di colui che ha acquisito conoscenze, abilità, autorevolezza, capacità di rispondere in maniera adeguata a situazioni complesse e che è quindi in grado di assumersi la responsabilità di una prestazione "difficile".

Il secondo punto da considerare riguarda il benessere e la soddisfazione del paziente che di solito si aspetta una sola cosa: provare il minimo dolore. Questo può essere in palese contrasto con l'applicazione dei principi scientifici su cui si fondano i protocolli prima considerati che prevedono, sempre nel caso specifico, uno o più criteri di rotazione della sede di puntura, al fine di prevenire complicanze quali la degenerazione dei tessuti, ma che in pratica provocano una sensazione dolorosa più accentuata nel paziente. Che fare allora? Secondo Spinsanti ${ }^{1}$ si tratta di un tipico caso

\footnotetext{
${ }^{1}$ Spinsanti S. Qualità dei servizi e soddisfazione dell'utente. Prospettive sociali e sanitarie, n.12/1995. L'autore individua un "quadrilatero della soddisfazione" entro cui può collocarsi la prestazione, in base alla quale il paziente può dichiararsi giustamente/ingiustamente soddisfatto oppure giustamente/ingiustamente insoddisfatto.
} 
in cui i "corni del dilemma" sono rappresentati da una parte da un paziente giustamente insoddisfatto (puntura eseguita secondo metodologia scientifica senza tener conto del suo punto di vista), dall'altra da un paziente ingiustamente soddisfatto (puntura eseguita dove il paziente lo desidera perché gli provoca il minor disagio).

Da questa contrapposizione hanno origine problemi, frizioni e conflitti. Trovare una giusta misura, sostenibile, rispettando ambedue i principi è impresa ardua. Occorre in ogni caso un costante atteggiamento interlocutorio, utilizzando in maniera appropriata lo strumento della negoziazione. Non si tratta di rinunciare aprioristicamente ai principi a favore di un quieto vivere, piuttosto di assicurare il meglio sul piano della relazione principi/benessere. Se un paziente tende insistentemente a condizionare l'infermiere nella scelta della zona di puntura a lui gradita, una ragionevole misura di compromesso potrebbe essere del tipo "una volta decidi tu, una volta decido io", tanto più perseguibile quanto la "personalità" professionale dell'infermiere sarà ben strutturata con conoscenze, abilità, atteggiamenti da assumere.

Avremmo in questo caso applicato il principio scientifico, a fronte di un paziente soddisfatto, nel $50 \%$ dei casi. Uno standard probabilmente insoddisfacente sui tempi lunghi, ma pur sempre un buon inizio.

elianto@tmn.it 